\title{
Stability evaluation of a DC micro-grid and future interconnection to an AC system
}

\author{
Santiago Sanchez ${ }^{\mathrm{a}, *}$, Marta Molinas ${ }^{\mathrm{a}}$, Marco Degano ${ }^{\mathrm{a}, \mathrm{b}}$, Pericle Zanchetta ${ }^{\mathrm{b}}$ \\ ${ }^{a}$ Department of Electrical Power Engineering, Norwegian University of Science and Technology, Trondheim NO 7491, Norway \\ ${ }^{\mathrm{b}}$ Department of Electrical and Electronic Engineering, University of Nottingham, University Park, Nottingham, UK
}

\begin{abstract}
A B S T R A C T
This paper presents the stability analysis of a DC micro-grid fed by renewable sources and the future interconnection with an AC micro-grid. This interconnection is realized through a voltage source converter, and the operation of the micro-grid is in island mode. The stability is analyzed by the Nyquist criteria with the impedance relation method. The frequency response of the models was obtained by the injection of a perturbation current at the operation point. Where this perturbation was at the input of the converter used to export power from the DC grid. Other perturbation was applied at the node of the micro-grid to evaluate its impedance. Finally the simulations show the impedance representation of the systems, and the stability for the interconnection of them. The experimental verification shows the impedance of the converter with the same tendency as the representation obtained by the analytical and simulation.
\end{abstract}

\section{Introduction}

The stable behavior is one of the most important factors in power systems. Its correct analysis ensures to the designer or operator that the system is going to be under normal conditions. Small signal techniques were developed for classical power systems, where generator to load current flow is always assumed [1]. But, future grids can present a system with power injection into the grid from the side of the costumer. These future grids will exhibit more nonlinear behavior and difficult coordination than the classical power system. In the case of DC distributed power electronics systems used in telecommunications, aircraft or ships the stability had been studied in Refs. [2-5]. Where stable region operation was identified. These methods employ frequency response and Nyquist criteria to present stability analysis. Linearization has been done, due to its simplicity and success record $[6,7]$. The wide spread choice to predict instability by the application of small signal analysis such as the Nyquist criterion or eigenvalues in AC systems with power electronics has been presented in Refs. [1,8-14]. However, it is necessary to analyze the stability of the distribution system with one of its prominent topology, in this work it is presented a variant of the CIGRE AC benchmark [15] for a DC residential low voltage micro-grid, and its stability has been studied at one of the power exchange nodes.

The paper is organized as follows: the Section 2 describes the modeling by impedance representation, Section 3 presents the grid structure and the method employed to control the DC voltage, and Section 4 describes the numerical results obtained from the simulations.

\section{Impedance representation methods}

The impedance is determined by the application of an ideal source with a frequency component. This source is a sinusoidal current or voltage type ( $i_{\mathrm{p}}$ or $v_{\mathrm{p}}$ respectively), and is denominated the perturbation. The magnitude has to be small with respect to the nominal operation value of the system. The frequency is chosen as $f_{\mathrm{p}}$ varying in a range. The input voltage and current are measured at the DC side of the grid or the VSC. Their Fourier transform is computed. Finally the values at the perturbation frequency determine the input impedance of the inverter for small signal stability analysis or output impedance of the grid $[2,8,9]$. The current or voltage injection to obtain the impedance for the VSC is presented in Fig. 1. The stability is analyzed with the Nyquist criteria used in Refs. $[14,12]$. Where the full system is partitioned into a source and a load subsystem, described by a Thevenin equivalent system in the case in which the source is considered 


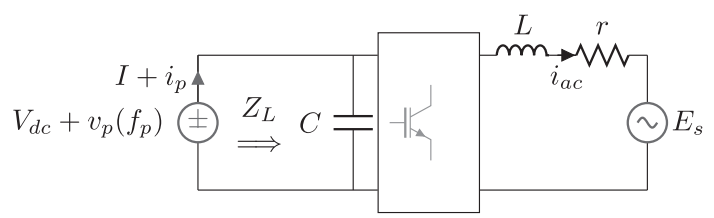

Fig. 1. Perturbation source for impedance measurement.

voltage type and a Norton equivalent system in which the source is considered current type, as is shown in Fig. 2. For voltage source type the analysis has to be realized over the Thevenin circuit and the voltage applied to the load subsystem is calculated in (1), where $V_{\mathrm{L}}$ is the voltage in the load, $Z_{\mathrm{S}}$ is the output impedance of the source, and $Z_{\mathrm{L}}$ is the input impedance of the load.

$V_{\mathrm{L}}=\frac{1}{1+{ }_{Z_{\mathrm{L}}}^{Z_{\mathrm{L}}}} V_{\mathrm{s}}$

The relation $Z_{\mathrm{S}} / Z_{\mathrm{L}}$ is used to realize the stability analysis, if its Nyquist plot counterclockwise encircles the point $-1+j 0$ then the system has an instability. And in case of use the Norton equivalent circuit the current in the load $I_{\mathrm{L}}$ is $(2)$, where $I_{\mathrm{S}}$ is the source current.

$I_{\mathrm{L}}=\frac{1}{1+{ }_{Z_{\mathrm{S}}}^{Z_{\mathrm{L}}}} I_{\mathrm{S}}$

\subsection{Voltage source converter}

The system used to interconnect the two grids is a power electronics three phase voltage source converter (VSC). This device operates as a rectifier and is used to link the two systems. The equations of a voltage source converter connected at the DC side to a grid and to an ideal AC system are described from (3)-(6).

$\frac{\mathrm{d} i_{d}}{\mathrm{~d} t}=\frac{E_{s d}-v_{d}+w L i_{q}-r i_{d}}{L}$

$\frac{\mathrm{d} i_{q}}{\mathrm{~d} t}=\frac{E_{s q}-v_{q}-w L i_{d}-r i_{q}}{L}$

$\frac{\mathrm{d} v_{\mathrm{dc}}}{\mathrm{d} t}=\frac{i_{\mathrm{dc}}+i_{\text {grid }}}{C}$

$i_{\mathrm{dc}}=s_{g d} i_{d}+s_{g q} i_{q}$

where $E_{s, k}$ with $k \in\{d, q\}$ is the voltage of the AC grid in the direct and quadrature axis, respectively. The current in the filter inductance $L$ is $i_{k}$. The voltage at the switch terminals of the converter is $v_{k}$. The DC voltage is $v_{\mathrm{dc}}$, the current at the DC side of the converter is $i_{\mathrm{dc}}$, the switching commands are $s_{g k}$, and the current flowing from the DC grid is $i_{\text {grid. }}$. The controller for this system is design with the set of equations from (7) to (11).

$v_{d}=-k_{p}\left(i_{\text {ref } d}-i_{d}\right)-k_{i} \gamma_{d}+w L i_{q}-\alpha e^{-\alpha t} E_{s} d$

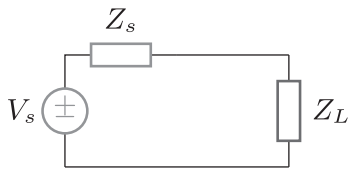

(a)

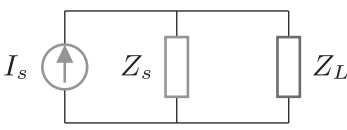

(b)
Fig. 2. Equivalent circuits representation to stability, (a) Thevenin, and (b) Norton.

$$
\begin{aligned}
& v_{q}=-k_{p}\left(i_{\text {ref } q}-i_{q}\right)-k_{i} \gamma_{q}-w L i_{d}-\alpha e^{-\alpha t} E_{s q} \\
& \dot{\gamma}_{d}=i_{\text {ref } d}-i_{d} \\
& \dot{\gamma}_{q}=i_{\text {ref } q}-i_{q}
\end{aligned}
$$

$s_{g k}=\frac{v_{k}}{v_{\mathrm{dc}}}$

The impedance of the load or converter connected to the grid can be estimated by the use of three techniques. They are described in the followed subsections.

\subsection{State space linearization}

The nonlinear function (12) can be linearized around an operation point and the system described by (13) and (14). For the converter the low frequency impedance is obtained by the method described in Ref. [14], the current of the source (or DC grid at the node) is the input to the system, the states are the AC currents, and the voltage in the DC capacitor; that is chosen as the output of the system. The matrix form of the differential equations for the inverter is (3) and (6).

$\dot{x}=f(x, u)$

$\Delta \dot{x}=A \Delta x+B \Delta u$

$y=C_{\mathrm{m}} \Delta_{\chi}$

For this method the input is equal to the grid current $u=i_{\text {grid, }}$, the states are $x=\left(i_{d}, i_{q}, v_{\mathrm{dc}}, \gamma_{d}, \gamma_{q}\right)^{T}$. The impedance in the frequency domain is calculated with (15).

$Z_{\mathrm{dc}}(s)=C_{\mathrm{m}}(s I-A)^{-1} B$

The impedance obtained by this method is:

$Z_{\mathrm{dc}}(s)=\frac{(s+\alpha) v_{\mathrm{dc}}^{2}}{s^{2} C v_{\mathrm{dc}}^{2}+s C v_{\mathrm{dc}}^{2} \alpha-i_{d} k_{i} \gamma_{d}(s+\alpha)+i_{d} \alpha E s d}$

\subsection{Linearization and transfer functions relation}

To obtain the impedance representation by this method the steps used in Refs. [16,17], are described to be applied over the model of the set (3)-(11). First the models are obtained in the time domain. In the second step, the Laplace transform is realized for the states in order to obtain the transfer functions. A linearization around the operation is required in this step. The transfer function that represents the impedance is obtained by the relation between the variables $v_{\mathrm{dc}}$ and $i_{\mathrm{dc}}$ in Laplace domain. And the impedance of the full converter is obtained by the equivalent parallel between $\mathrm{DC}$ capacitance and the impedance of the converter. The obtained impedance for this system is presented from (17) and (18), and the final model is shown in (19).

$\left.Z_{\mathrm{vsc}}(s)=-\frac{v_{\mathrm{dc}}}{i_{\mathrm{dc}}} \frac{v_{\mathrm{dc}}(r+s L)^{2}(s+\alpha)^{2}}{a_{4} s^{4}+a_{3} s^{3}+a_{2} s^{2}+a_{1} s+a_{0}}\right)$

$a_{k}=f_{k}\left(K_{\text {ptrans }}, G_{\mathrm{a}}, x_{0}\right) ;(k \in\{0, \ldots 4\}$

$Z_{\mathrm{dc}}(s)=\frac{Z_{\mathrm{C}}(s) Z_{\mathrm{vsc}}(s)}{Z_{\mathrm{C}}(s)+Z_{\mathrm{vsc}}(s)}$ 
where $K_{\text {ptrans }}$ is the gain of the power from the $a b c$ to the $d q$ reference frame, $G_{\mathrm{a}}=1 / V_{\mathrm{dc}}$ at the nominal value, the parameters $a_{k}$ are described in the Appendix $A, Z_{C}$ is the impedance of the $D C$ capacitor.

\subsection{Harmonic balance principle}

The harmonic balance $[18,19]$ is used to obtain the impedance by injecting a perturbation into the converter at the DC side and see its effect at the AC side. The approximation is obtained by the assumption employed in Refs. [20,21], which use no energy stored in the filters of the DC and AC side, it can be seen in (20).

$v_{\mathrm{dc}}(t) i_{\mathrm{dc}}(t)=v_{a}(t) i_{a}(t)+v_{b}(t) i_{b}(t)+v_{c}(t) i_{c}(t)$

where $v_{a, b, c}(t), i_{a, b, c}(t)$ are the voltage, and current for the AC side respectively, and $v_{\mathrm{dc}}(t)$ is presented as a time variable signal like (21) in order to include a sinusoidal perturbation voltage applied from the DC side to obtain the small signal impedance. Then, the current $i_{\mathrm{dc}}(t)$ at the DC side is expected to be time variable.

$v_{\mathrm{dc}}(t)=V_{\mathrm{d}}+V_{\mathrm{p}} \cos \left(\omega^{\mathrm{p}} t\right)$

where $V_{\mathrm{d}}, V_{\mathrm{p}}$ are the constant component, and the perturbation maximal value, $\omega_{\mathrm{p}}$ is the angular velocity of the perturbation signal.

In order to evaluate the left hand side of (20), the time variable DC current is assumed as in (22) with a constant component $I_{\mathrm{d}}$, a perturbation value $I_{\mathrm{p}}$, and a lagged angle $\varphi_{\mathrm{p}}$.

$i_{\mathrm{d}}(t)=I_{\mathrm{d}}+I_{\mathrm{p}} \cos \left(\omega_{\mathrm{p}} t-\phi \mathrm{p}\right)$

The evaluation of (21), and (22) in the left hand side of (20) is presented in (23).

$$
\begin{aligned}
p_{\mathrm{dc}}(t)= & I_{\mathrm{d}} V_{\mathrm{d}}+\frac{1}{2} I_{\mathrm{p}} V_{\mathrm{p}} \cos \left(\phi_{\mathrm{p}}\right)+I_{\mathrm{d}} V_{\mathrm{p}} \cos \left(\omega^{\mathrm{p}} t\right)+ \\
& I_{\mathrm{p}} V_{\mathrm{d}} \cos \left(\omega_{\mathrm{p}} t-\phi_{\mathrm{d}}\right)+\frac{1}{2} I_{\mathrm{p}} V_{\mathrm{p}} \cos \left(2 \omega_{\mathrm{p}} t-\phi \mathrm{d}\right)
\end{aligned}
$$

The PWM signals are obtained by comparing a triangular waveform $v_{\text {tri }}(t)$ with peak value $V_{\text {tri }}$ and the control voltages $v_{C k}(t)=V_{C} \cos \left(\omega_{\mathrm{e}} t-\gamma_{k}\right)$ in which $k=\{a, b, c\}$ and $\gamma_{k}=\{0,120,240\}$ degrees for its respective phase; where the duty ratios in the average representation can be written as is shown in (24) [21].

$d_{k}(t)=0.5+0.5 \frac{V_{\mathrm{C}}}{V_{\mathrm{tri}}} \cos \left(\omega_{\mathrm{e}} t-\gamma k\right)$

Using the duty ratios of (24) and the time variable DC voltage to obtain the phase to DC neutral voltages (25), and (26) are obtained.

$$
\begin{aligned}
v_{k N}(t)= & d_{k}(t) v_{\mathrm{dc}}(t) \\
v_{k N}(t)= & \frac{V_{\mathrm{d}}}{2}+\frac{V_{\mathrm{d}} V_{\mathrm{C}}}{2} V_{\text {tri }} \cos \left(\omega_{\mathrm{e}} t-\gamma_{k}\right)+\frac{V_{\mathrm{p}}}{2} \cos \left(\omega^{\mathrm{p}} t\right) \\
& +\frac{V_{\mathrm{p}} V_{\mathrm{C}}}{8 V_{\text {tri }}}\left[2 \cos \left(\left(w_{\mathrm{e}}+w_{\mathrm{p}}\right) t-\gamma_{k}\right)\right. \\
& \left.+2 \cos \left(\left(w_{\mathrm{e}}-w_{\mathrm{p}}\right) t-\gamma_{k}\right)\right]
\end{aligned}
$$

The terms $0.5 V_{\mathrm{d}}$, and $0.5 V_{\mathrm{p}} \cos \left(\omega_{\mathrm{p}} t\right)$ in (26) are offset voltages, and in each phase are considered as zero-sequence voltages as is analyzed in Ref. [21] for the term $0.5 V_{\mathrm{d}}$, therefore do not produce current flow in the balanced three phase load. Then the average phase to neutral voltages for the balanced star load with neutral $n$, are as in (27).

$$
\begin{aligned}
v_{k n}(t)= & \frac{V_{\mathrm{d}} V_{\mathrm{C}}}{2} V_{\text {tri }}^{-} \cos \left(\omega_{\mathrm{e}} t-\gamma_{k}\right)+\frac{V_{\mathrm{p}} V_{\mathrm{C}}}{4 V_{\text {tri }}}\left[\cos \left(\left(w_{\mathrm{e}}+w_{\mathrm{p}}\right) t-\gamma_{k}\right)\right. \\
& \left.+\cos \left(\left(w_{\mathrm{e}}-w_{\mathrm{p}}\right) t-\gamma_{k}\right)\right]
\end{aligned}
$$

In (27) there are three frequency components and each signal has its respective maximum value. To facilitate the use of (27), it is written as (28), where $V_{1} \neq V_{2}$, and $V_{2}=V_{3}$.

$$
\begin{aligned}
v_{k n}(t)= & V_{1} \cos \left(\omega_{\mathrm{e}} t-\gamma_{k}\right)+V_{2} \cos \left(\left(w_{\mathrm{e}}+w_{\mathrm{p}}\right) t-\gamma_{k}\right) \\
& +V_{3} \cos \left(\left(w_{\mathrm{e}}-w_{\mathrm{p}}\right) t-\gamma_{k}\right)
\end{aligned}
$$

Assuming that the system has a linear part the currents in each phase are expected to have the same frequency components that the voltages, and its respective lagged load angle. Also, the magnitude of the current in its respective frequency is assumed to be proportional to the magnitude of the impedance at the same frequency. Hence the current in each phase is (29).

$$
\begin{aligned}
i_{k n}(t)= & I_{1} \cos \left(\omega_{\mathrm{e}} t-\gamma_{k}-\phi_{1}\right)+I_{2} \cos \left(\left(w_{\mathrm{e}}+w_{\mathrm{p}}\right) t-\gamma_{k}-\phi 2\right) \\
& +I_{3} \cos \left(\left(w_{\mathrm{e}}-w_{\mathrm{p}}\right) t-\gamma_{k}-\phi_{3}\right)
\end{aligned}
$$

The magnitudes $I_{1}, I_{2}$, and $I_{3}$ in (29) are functions of the three phase balanced impedance that is a function of the frequency, and is notated as $Z_{1}\left(\omega_{\mathrm{e}}\right), Z_{2}\left(\omega_{\mathrm{e}}+\omega_{\mathrm{p}}\right)$, and $Z_{3}\left(\omega_{\mathrm{e}}-\omega_{\mathrm{p}}\right)$. Therefore, the angles of the load are $\phi_{1}\left(\omega_{\mathrm{e}}\right), \phi_{2}\left(\omega_{\mathrm{e}}+\omega_{\mathrm{p}}\right)$, and $\phi_{3}\left(\omega_{\mathrm{e}}-\omega_{\mathrm{p}}\right)$.

The controller can be added assuming that there is a PI regulator in the converter, the reference current can be model by $i_{\text {refk }}=I_{\text {reff }} \cos \left(\omega t-\phi_{1}-\gamma_{k}\right)$, the integrated reference current is $\int i_{\text {ref } k}=\left(I_{\text {ref }} / \omega\right) \sin \left(\omega t-\phi_{1}-\gamma k\right)$. The controller is applied into refk the variable $V_{C}$ for each phase as follows:

$V_{\mathrm{Ck}}=k_{p}\left(i_{\text {ref } k}-i_{k n}\right)+k_{i} \int\left(i_{\text {ref } k}-i_{k n}\right) \mathrm{d} t$

Equations (28)-(30) are used in the right hand side of (20) to obtain the AC power (31) at the injected perturbation frequency and to build the model that describes the impedance.

$p_{\mathrm{ac}}\left(w_{\mathrm{p}} t\right)=K_{\mathrm{pac}}\left(k_{\mathrm{p}} f_{1 \text { power }}\left(\omega_{\mathrm{p}} t\right)+k_{\mathrm{i}} f_{2 \text { power }}\left(\omega^{\mathrm{p}} t\right)\right)$

where $K_{\mathrm{pac}}$ is the gain of the power obtained. The functions $f_{l \text { power }}\left(\omega_{\mathrm{p}}\right)$ with $l \in\{1,2\}$ describe the power functions obtained for the proportional and integral controller, respectively. The equation (31) is compare with the fourth term of (23) in order to obtain an expression for $i_{\mathrm{p}}$. Then, to find the value of $I_{\mathrm{p}}$ as a function of the AC impedance in frequency a complex notation is used in (32)-(38).

$$
\begin{aligned}
& \boldsymbol{I}_{\mathrm{p}, k_{\mathrm{p}}}=k_{\mathrm{p}}\left(\mathbf{I}_{3}\left(w_{\mathrm{e}} w_{\mathrm{p}}^{2}-w_{\mathrm{e}}^{3}\right)+\boldsymbol{I}_{2}\left(w_{\mathrm{e}} w_{\mathrm{p}}^{2}-w_{\mathrm{e}}^{3}\right)\right) I_{1} e^{-j \phi_{1}} \\
& \boldsymbol{I}_{\mathrm{p}, k_{i}}=k_{i}\left(\boldsymbol{I}_{3 b}\left(-w_{\mathrm{e}} w_{\mathrm{p}}-w_{\mathrm{e}}^{2}\right)+\boldsymbol{I}_{2 b}\left(w_{\mathrm{e}} w_{\mathrm{p}}-w_{\mathrm{e}}^{2}\right)\right) I_{1} e_{-j}\left(\phi_{1}+\pi / 2\right)
\end{aligned}
$$

$\boldsymbol{I}_{2}=I_{2} e^{-j \phi_{2}}$

$\boldsymbol{I}_{2 b}=\boldsymbol{I}_{2} e^{-j\left(\phi_{2}+\pi / 2\right)}$

$\mathbf{I}_{3}=I_{3} e^{-j \phi_{3}}$

$\mathbf{I}_{3 b}=\boldsymbol{I}_{3} e^{-j\left(\phi_{3}+\pi / 2\right)}$ 


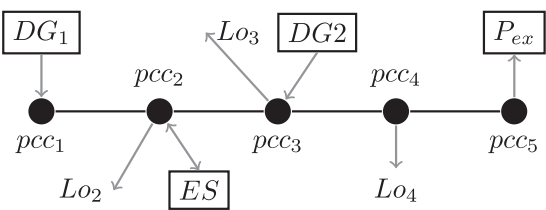

Fig. 3. DC micro-grid used for the stability test.

$\boldsymbol{I}_{\mathrm{p}}=\frac{\left(\boldsymbol{I}_{\mathrm{p}, k_{\mathrm{p}}}+\boldsymbol{I}_{\mathrm{p}, k_{i}}\right)}{V_{\mathrm{tri}}\left(w_{\mathrm{e}}^{3}-w_{\mathrm{e}} w_{\beta}\right)}$

The next step is to compute the impedance by the relation presented in (39).

$\boldsymbol{Z}_{\mathrm{dc}}(\omega)=\frac{\boldsymbol{V}_{\mathrm{p}}(\omega)}{\boldsymbol{I}_{\mathrm{p}}(\omega)}$

Finally, the complete converter impedance is found by the parallel of the capacitor and (39).

\section{Grid topology}

The topology described in Fig. 3 is based on a segment of the CIGRE benchmark low voltage micro-grid [15], with some modification to operate with direct current, and a voltage of $400 \mathrm{~V}$ DC. The line parameters are calculated for a service connection feeder $16 \mathrm{~mm}^{2} \mathrm{Cu}$. Also, they are described in Table $1, \operatorname{Load}_{i}$ represents the load in the node $i$, the line between the nodes is Line ${ }_{(i-1)-i}$, and its resistance and inductance values are $r$ and $L$, respectively. The distance between each node is $30 \mathrm{~m}$. The system is composed by a wind turbine (WT) with $5 \mathrm{~kW}$ of power $\left(\mathrm{DG}_{1}\right)$, with a permanent magnet generator, a diode bridge rectifier, followed the $\mathrm{DC}-\mathrm{DC}$ converter. The second renewable source $\left(D_{2}\right)$ is a photo-voltaic (PV) $5 \mathrm{~kW}$ system operating in maximum power extraction and a DC-DC converter to regulate the voltage supplied to the grid. The third distributed generator is a battery bank (ES) which contributes in the power sharing, and is coupled to the grid by a DC-DC bidirectional converter. The power can be exported or imported by a voltage source converter $\left(P_{\mathrm{ex}}\right)$ connected to an AC system. Where the coordination of the grid has been realized with power sharing by drop control $P-V$, and one operation point is used for the stability test.

\subsection{Wind turbine model}

The small power turbine is modeled and controlled as in Ref. [22]. A description of the dynamic system is described in the followed subsections.

\subsubsection{Wind turbine}

A simplified model that shows the relation between the wind velocity, the generated power $\left(P_{\mathrm{m}}\right)$ and rotational speed $(\omega)$ is described in (40).

Table 1

Line parameters for the micro-grid structure.

\begin{tabular}{ll}
\hline Parameter [m] & Value \\
\hline Line $_{(i-1)-i}$ & $r_{(i-1)-i}=0.041 \Omega, L_{(i-1)-i}=7.83 \mu \mathrm{H}$ \\
Load $_{2}$ & $48 \Omega(3.33 \mathrm{~kW})$ \\
Load $_{3}$ & $320 \Omega(0.5 \mathrm{~kW})$ \\
Load $_{4}$ & $160.0 \Omega(1 \mathrm{~kW})$ \\
\hline
\end{tabular}

$P_{\mathrm{m}}=\frac{1}{2} \rho C_{\mathrm{p}}(\lambda, \beta) A_{\mathrm{w}} V_{\mathrm{w}}^{3}$

where $\rho$ is the air density, $\beta$ is the pitch angle which is chosen as zero for small power turbines, $A_{\mathrm{W}}$ the area covered by turbine, $V_{\mathrm{w}}$ is the wind speed, $\lambda$ is the tip speed ratio defined in (41), $\omega_{\mathrm{r}}$ rotor angular velocity, and $\Phi$ is the turbine blades diameter.

$\lambda=\frac{\omega_{\mathrm{r}}}{V_{\mathrm{w}}}\left(\begin{array}{l}\Phi \\ 2\end{array}\right)$

$C_{\mathrm{p}}(\lambda)=0.5\left(\begin{array}{c}116 \\ \lambda_{i}\end{array}-5\right) e^{\frac{-21}{\lambda_{i}}}+0.01 \lambda$

with $\lambda_{i}$ described in (43).

$\lambda_{i}=\left(\begin{array}{l}4 \\ \lambda\end{array}-0.03\right)^{-1}$

A one mass model describes the dynamics of the mechanical speed (44).

$\frac{\mathrm{d} \omega \mathrm{r}}{\mathrm{d} t}=\frac{1}{J_{\mathrm{m}}}\left(T_{\mathrm{m}}-T_{\mathrm{E}}\right)$

where $J_{\mathrm{m}}$ is the electrical machine and turbine inertia coefficient, $T_{\mathrm{E}}$ electrical torque and the mechanical torque is defined as (45).

$T_{\mathrm{m}}=\frac{P_{\mathrm{m}}}{\omega_{\mathrm{r}}}$

\subsubsection{Permanent magnet synchronous generator}

A model in the space vector representation is presented in this work [23], the system is described in (46)-(48) as generator operation. The parameters of the machine are $n_{\mathrm{p}}$ the pole pairs number, $L_{\mathrm{s}}, R_{\mathrm{S}}$ the stator inductance and resistance respectively, $J$ the inertia coefficient of the machine, $\boldsymbol{i}_{\mathrm{s}}, \boldsymbol{v}_{\mathrm{s}}$ the stator current and voltage respectively, $\Phi_{\mathrm{m}}$ magnetization flux, and $\theta_{\mathrm{r}}$ rotor angle.

$L_{\mathrm{s}} \frac{\mathrm{d}}{\mathrm{d} t} \boldsymbol{i}_{\mathrm{s}}=-R_{\mathrm{s}} \boldsymbol{i}_{\mathrm{s}}+j n_{\mathrm{p}} \omega_{\mathrm{r}} \Phi_{\mathrm{m}} e^{j n_{\mathrm{p}} \theta_{\mathrm{r}}}-\boldsymbol{v}_{\mathrm{s}}$

$J \frac{\mathrm{d}}{\mathrm{d} t} \omega_{\mathrm{r}}=T_{\mathrm{m}}-\Phi_{\mathrm{m}}\left[I_{\mathrm{mag}}\left\{\boldsymbol{i}_{\mathrm{s}} e^{-j n_{\mathrm{p}} \theta_{\mathrm{r}}}\right\}\right]$

$\frac{\mathrm{d}}{\mathrm{d} t} \theta_{\mathrm{r}}=\omega_{\mathrm{r}}$

where $I_{\text {mag }}\{\}$ is the imaginary part, the space vector notation for the stator current and voltage as described in (49) and (50), with $\gamma_{k}=\{0,2 \pi / 3,4 \pi / 3\}, i_{k}(t)$, and $v_{k}(t)$ the phase current, and voltage respectively.

$\boldsymbol{i}_{\mathrm{s}}=\sum_{k=a}^{c} i_{k}(t) e^{j \gamma_{k}}$

$\boldsymbol{v}_{\mathrm{s}}=\sum_{k=a}^{c} v_{k}(t) e^{j \gamma_{k}}$

The machine can be seen as a source in series with a resistance and an inductance in the stator, it can be observed in Fig. 4. 


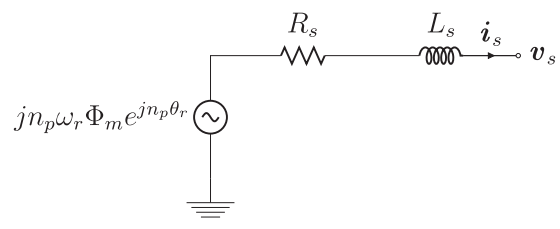

Fig. 4. Permanent magnet machine equivalent circuit.

\subsubsection{Full bridge diode rectifier}

The arrangement of the rectifier is a full bridge, six pulse, and three phase, with a filter capacitor at the DC side. The model description assumes the inductance value at its AC terminals to be zero [20], the instantaneous DC voltage $v_{\mathrm{dc}}$ is presented in (51), where $V_{\mathrm{LL}}$ is the rms line to line voltage value. The direct current $I_{\mathrm{dc}}$ in function of the peak phase current $I_{\mathrm{m}}$ is (52).

$v_{\mathrm{dc}}(t)=\sqrt{2} V_{\mathrm{LL}} \cos \omega t \quad-\frac{\pi}{6}<\omega t<\frac{\pi}{6}$

$I_{\mathrm{dc}}=\frac{\sqrt{3}}{2} I_{\mathrm{m}}$

For the rectifier the DC voltage average value $V_{\mathrm{dc}}$ is (53).

$V_{\mathrm{dc}}=\frac{3}{\pi} \sqrt{2} V_{\mathrm{LL}}$

\subsubsection{Buck-boost converter model}

The Buck-boost structure is chosen for this work due to its operation characteristics. Where the input voltage can be higher or lower than the output voltage. Continuous conduction mode is assumed for the design of the model. In (54) the dynamic of the inductor current $\left(i_{\mathrm{L} 1}\right)$ is presented, with a switching signal $q_{1}$, the inductance is $L_{1}$, and the source voltage $v_{\text {inp1 }}$. The dynamic voltage of the capacitance $\left(v_{01}\right)$ is in (55), the capacitance of the converter is $C_{\mathrm{f} 1}$, the parameter $i_{01}$ is output current.

$\frac{\mathrm{d} i_{\mathrm{L} 1}}{\mathrm{~d} t}=\frac{\left(q_{1}-1\right) v_{\mathrm{o} 1}+q_{1} v_{\text {inp } 1}}{L_{1}}$

$\frac{\mathrm{d} v_{\mathrm{o} 1}}{\mathrm{~d} t}=\frac{\left(1-q_{1}\right) i_{\mathrm{L} 1}-i_{\mathrm{o} 1}}{C_{\mathrm{f} 1}}$

The DC-DC structure is presented in Fig. 5. An ideal switch $(S)$ is showed in the place of the IGBT [21], and the load resistance is $R_{\mathrm{bb}}$.

\subsection{Solar module}

The equations that describe the solar cell are described in Refs. $[24,25]$. The dynamic is represented from (56) to (62).

$i=n_{\mathrm{p}} I_{\mathrm{ph}}-n_{\mathrm{p}} i_{\mathrm{d}}-n_{\mathrm{p}} i_{\mathrm{r}}$

$i_{\mathrm{ph}}=I_{\mathrm{SCO}} \frac{S}{S_{0}}+C_{t}\left(T-T_{\mathrm{ref}}\right)$

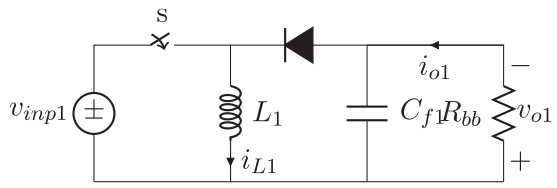

Fig. 5. DC-DC Buck-boost converter.
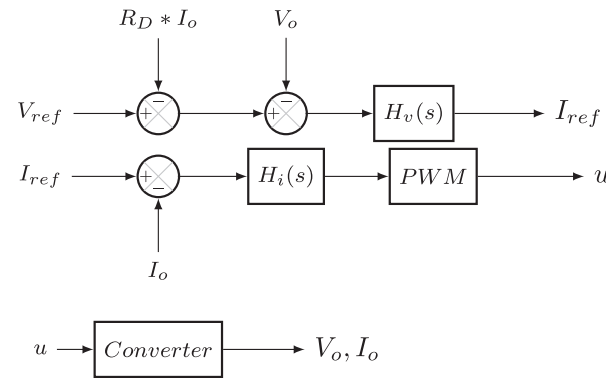

Fig. 6. Primary control method

$i_{\mathrm{d}}=I_{0}\left(e^{\frac{q v_{\mathrm{sh}}}{A k T}}-1\right)$

$\left.I_{0}=I_{\mathrm{S} 0} \frac{T}{T_{\text {ref }}}\right)^{3} e^{\frac{q E g}{A k}\left(\frac{1}{T_{\text {ref }}}-\frac{1}{T}\right)}($

$i_{\mathrm{r}}=\frac{v_{\mathrm{sh}}}{R_{\mathrm{sh}}}$

$v_{\mathrm{sh}}=\frac{v}{n_{\mathrm{s}}}+i R_{\mathrm{se}}$

$T=T_{\mathrm{a}}+k_{\mathrm{s}} S$

where the system parameters are: $S_{0}$ is the standard light intensity, $T_{\text {ref }}$ the temperature under standard test conditions, $R_{\mathrm{sh}}$ and $R_{\mathrm{se}}$ are the shunt and series resistances in the solar cell, $I_{S C O}$ the short circuit current of each solar cell at $T_{\mathrm{ref}}$, $I_{\mathrm{s}}$ the diode saturation current of the cell at $T_{\mathrm{ref}}, E_{\mathrm{g}}$ is the band energy of the material, $A$ the ideal factor, $C_{\mathrm{t}}$ the temperature coefficient, the coefficient $k_{\mathrm{s}}$ that defines the light intensity disturbance, $v_{\text {sh }}$ voltage at the shunt resistor, the electron charge is $q$, the Boltzmann constant is $k, S$ is the light intensity, the ambient temperature is $T_{\mathrm{a}}, v$ is the voltage at the solar module terminals, and $i$ is the output current.

\subsection{Dynamic battery model}

The battery array is model in (63) and (64) [26]. The interconnection is realized by a bidirectional power electronic interface [24].

$\mathrm{SOC}=100\left(1-\frac{\int i_{\mathrm{b}} \mathrm{d} t}{Q}\right)($

$v_{\mathrm{b}}=V_{\mathrm{o}}+R_{\mathrm{b}} i_{\mathrm{b}}-K \frac{Q}{Q+\int i_{\mathrm{b}} \mathrm{d} t}+A e^{B} \int\left(i_{\mathrm{b}} \mathrm{d} t\right.$

where the parameters are: SOC is the state of charge, $v_{\mathrm{b}}$ is the terminal voltage, $V_{\mathrm{o}}$ is the open circuit voltage, $i_{\mathrm{b}}$ is the charging current, $Q$ is the capacity, $R_{\mathrm{b}}$ is the internal resistance, $A$ is the exponential zone voltage, $B$ is the exponential capacity, $K$ is the polarization voltage.

\section{4. $D C-D C$ converter control strategy for power sharing}

The converters used to keep the voltage constant in the DC micro-grid employ the strategy described in Ref. [27]. Fig. 6 

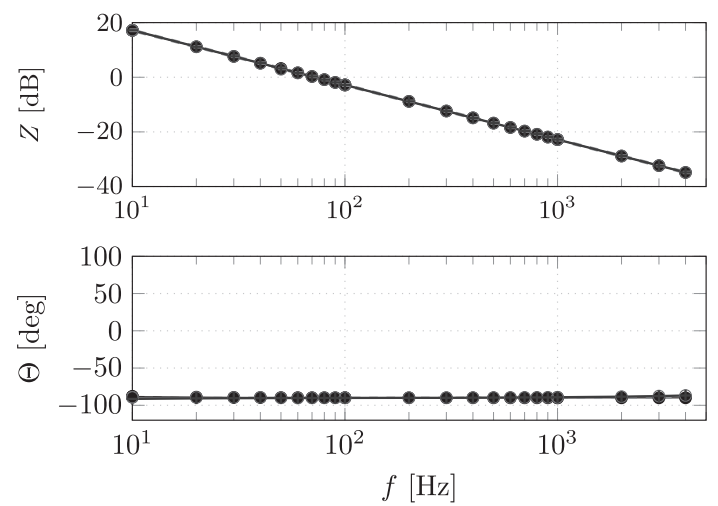

Fig. 7. Impedance for the VSC system operating at $5 \% P_{\mathrm{n}},\left({ }^{*}-\right)$ state space, $(--) \mathrm{TF},(\mathrm{O})$ harmonic balance, and (+-) simulation.

describes a primary controller with an inner loop to control the current injected to the system. And an outer loop to control the voltage in terminals of the converter. Hence, is assured the island mode operation and not communication between the sources is required. The grid operates under constant voltage at the point of common coupling with a primary power-voltage droop controller to realize power sharing [27]. The maximum voltage variation is defined as $\Delta V_{\mathrm{dc}}= \pm 4 \%$. The method is based on the measurement of the voltage and current in terminals of the converter. Where from Fig. $6, V_{\text {ref, }} I_{\text {ref }}$ are the voltage and current references respectively. $R_{\mathrm{D}}$ is a virtual output impedance, $H_{v}(s), H_{i}(s)$ are the voltage and current controllers, respectively. And $V_{0}, I_{0}$ are the voltage and current of the converter.

\section{Results}

The first database were created by simulation with the Simulink-Matlab software in order to validate and compare the models obtained by the analytical impedance representation. The symbolic manipulation of the equations was develop with the software Maple, Finally the database for the experimental verification were stored and manipulated to obtain the impedance with a Matlab script. Hence, the impedances for the VSC were obtained for each method, and the DC micro-grid was calculated by the injection of a DC perturbation current at node $\mathrm{pcc}_{5}$ in Fig. 3 plus the operation current that the system is going to have. The node will be used to interconnect the systems, where the converter in this case will export a power of $800 \mathrm{~W}$. Figs. 7 and 8 present the impedance behavior of each system.

The frequency analysis for the grid and the VSC impedance is realized with the Thevenin equivalent system. The relation is

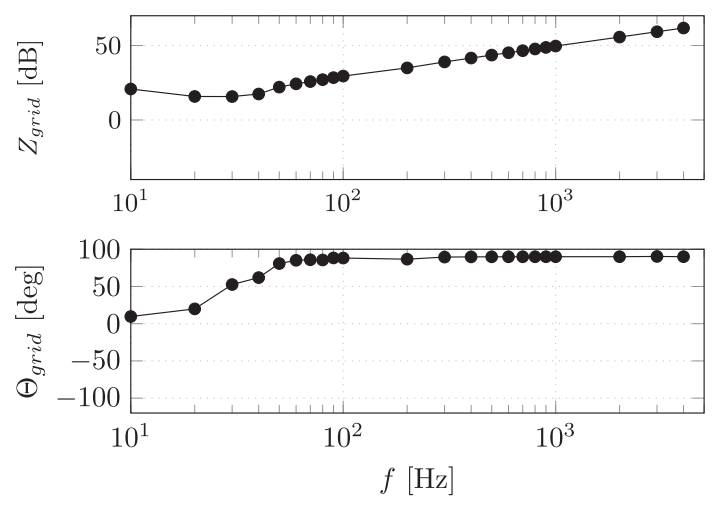

Fig. 8. Impedance for the DC micro-grid.
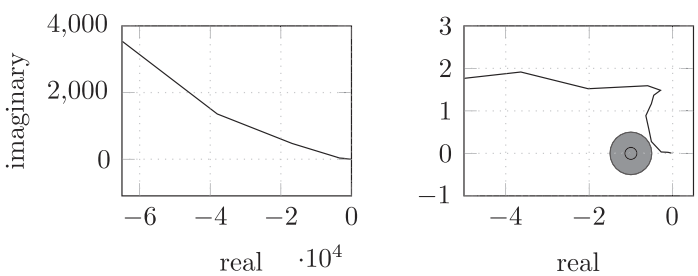

Fig. 9. Nyquist analysis for the future interconnection.

presented in Fig. 9. Where the stability margin of $6 \mathrm{~dB}$ and $30^{\circ}$ is considered in this work [2]. From Fig. 9 is clear that the stability margin is not reached for the frequency range of [10 $\left.-f_{\mathrm{sw}} / 2\right] \mathrm{Hz}$, where $f_{\mathrm{sw}}=10 \mathrm{kHz}$ is the switching frequency used in the VSC. During the tests of the VSC impedance, it is coupled to its AC passive impedance and the DC capacitor. Therefore, the impedance at the DC side is highly affected by the passive systems used [8]. Hence, with the result in Fig. 9 the system would keep a stable state when is coupled to the DC micro-grid. An improvement in the stability response can be reached by addition of passive filters, that can shape the final response as is described in Ref. [16] for an aircraft electric grid. The time domain tests are shown in Fig. 10. It presents the transient behavior of the complete grid for small disturbances. When the connection between the DC micro-grid and the AC system is set it can be seen the damping of the voltage restoration and slow variation in the power shared by the converters. At 1.0 s the connection is realized and the systems keep the stability producing a low drop (3\%) in the voltage of the grid $\left(v_{\text {grid }}\right)$, the operation voltage is recovered by the action of the droop controller implemented in the converters. The power shared is presented in Fig. 10, a condition is that the input power to the renewable energy is constant during the time interval studied. Where $p_{\mathrm{pv}}$ is the power developed by the solar energy system, $p_{\mathrm{wt}}$ is the power from the wind turbine, and $p_{\text {batt }}$ is the power from the batteries bank. Finally, the power drawn by the VSC used to interconnect the DC grid and the AC system is $p_{\mathrm{vsc}}$.

\subsection{Experimental verification}

The system used to obtain the experimental impedance is described in Fig. 11 and the test bench picture is shown in Fig. 12. The source $v_{\text {gen }}$ is the perturbation signal with variable frequency produced by a signal generator (1). This is isolated by a small power transformer and connected to a linear amplifier (2), hence the perturbation is applied to the DC grid by the secondary of the
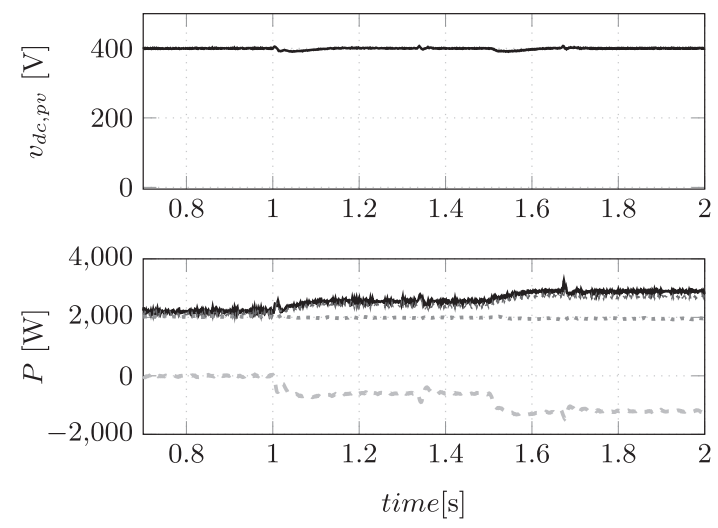

Fig. 10. DC grid interconnection with the $\mathrm{AC}$ system at $1.0 \mathrm{~s},-p_{\mathrm{pv}},-p_{\mathrm{wt}},-p_{\mathrm{batt}}$, and $-p_{\mathrm{vsc}}$. 


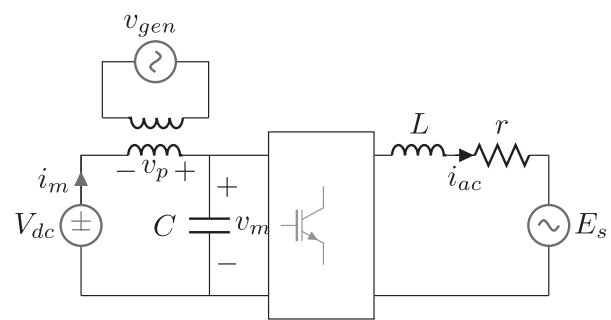

Fig. 11. System configuration to obtain the impedance of the VSC.

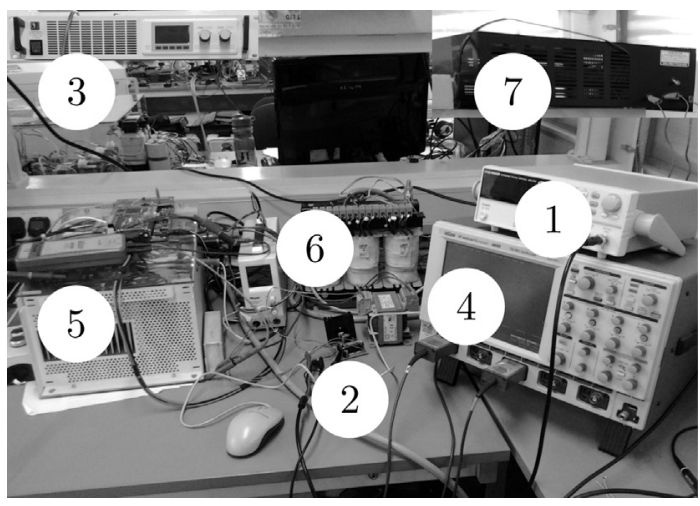

Fig. 12. Experimental test-bench used to obtain the impedance of the VSC system.

transformer $\left(v_{\mathrm{p}}\right)$. The $V_{\mathrm{dc}}$ is a programmable DC source (3), the impedance is obtained by the measures of the voltage $v_{\mathrm{m}}$ and the current $i_{\mathrm{m}}(4)$. The voltage source converter (5) has the capacitance coupled to the DC bar and cannot be removed, also is composed by a filter inductance with its respective resistance (6), and finally $E_{S}$ is the AC source connected (7).

The magnitude of the experimental impedance and angle is shown in Fig. 13, where the shape of the magnitude is correlated to the predicted by the analytical methods. However, the angle obtained with the physical system does not match the analytical angle for low frequency values (i.e. $f<20 \mathrm{~Hz}$ ). This problem can be related with some noise problems of the measures at the lower operation point employed. But, as can be seen from the experimental response and the analytical methods the main behavior of the angle is with negative values and a correlation has been found with the theory used to obtain the impedance.
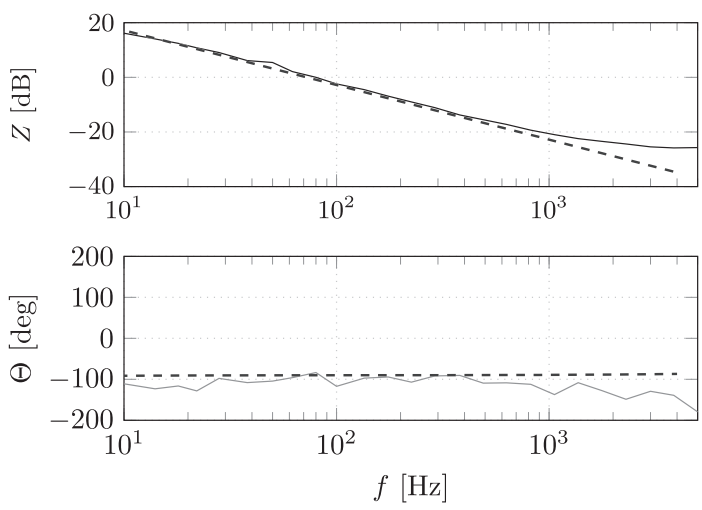

Fig. 13. Impedance magnitude and phase for the VSC system operating at $5 \% P_{n}$, experimental impedance (continuous), and the simulation (dashed).

\section{Conclusions}

The stability analysis of the future interconnection of a DC micro-grid and an AC system was developed. The Nyquist criteria with Thevenin equivalent system were realized. This equivalent was selected due to the behavior and control strategies employed by the converters used in the renewable grid. Where the outer loop was based on voltage control, and the converters have capacitors at the output (i.e. the voltage through them cannot vary suddenly).

As is seen in Fig. 9 using a low restrictive margin criteria, this analysis predicts the stability at the moment of coupling the $\mathrm{AC}$ system with the DC grid. Also, the small disturbances had been consider.

The impedance representation methods used and the stability study can be used with the passive filter design to improve the quality of the micro-grids.

\section{Appendix A. Parameters of the numerator for the transfer function method}

The parameters of the numerator in (17) are presented below, the function $G_{\text {is }}=k_{\mathrm{p}}+k_{i} / s$ describes the controller in the Laplace domain.

$$
\begin{aligned}
a_{4}= & i_{\mathrm{dc}} L^{2} \\
a_{3}= & K_{\mathrm{trans}} G_{\mathrm{a}}^{2} G_{\mathrm{is}}^{2} i_{\mathrm{ref} d}^{2} L v_{\mathrm{dc}}+K_{\mathrm{trans}} G_{\mathrm{a}} \alpha i_{\mathrm{ref} d}^{2} G_{\mathrm{is}} L^{2} \\
& -2 K_{\mathrm{trans}} G_{\mathrm{a}}^{2} E_{s d} G_{\mathrm{is}} i_{\mathrm{ref} d} L v_{\mathrm{dc}}+2 i_{\mathrm{dc}} L^{2} \alpha-K_{\mathrm{trans}} G_{\mathrm{a}} \alpha i_{\mathrm{ref} d} E_{s d} L^{2} \\
& +2 i_{\mathrm{dc}} r L+K_{\mathrm{trans}} G_{\mathrm{a}}^{2} E_{s d}^{2} L v_{\mathrm{dc}}
\end{aligned}
$$

$$
\begin{aligned}
a_{2}= & -2 K_{\text {trans }} G_{\mathrm{a}}^{2} E_{s d} G_{\mathrm{is}} \alpha i_{\mathrm{ref} d} L v_{\mathrm{dc}}+i_{\mathrm{dc}} L^{2} \alpha^{2}+4 i_{\mathrm{dc}} r L \alpha+i_{\mathrm{dc}} r^{2} \\
& -2 K_{\mathrm{trans}} G_{\mathrm{a}}^{2} E_{s d} G_{\mathrm{is}} i_{\mathrm{ref} d} r v_{\mathrm{dc}}-K_{\mathrm{trans}} G_{\mathrm{a}} \alpha_{2} i_{\mathrm{ref} d} E_{s d} L^{2} \\
& +K_{\mathrm{trans}} G_{\mathrm{a}}^{2} E_{s d}^{2} r v_{\mathrm{dc}}+2 K_{\mathrm{trans}} G_{\mathrm{a}} \alpha i_{\mathrm{ref} d}^{2} G_{\mathrm{is}} r L \\
& +K_{\text {trans }} G_{\mathrm{a}}^{2} G_{\mathrm{is}}^{2} i_{\mathrm{ref} d}^{2} r v_{\mathrm{dc}}-2 K_{\mathrm{trans}} G_{\mathrm{a}} \alpha i_{\text {ref } d} E_{s d} r L \\
& +2 K_{\mathrm{trans}} G_{\mathrm{a}}^{2} E_{s d}^{2} L v_{\mathrm{dc}} \alpha
\end{aligned}
$$

$$
\begin{aligned}
a_{1}= & K_{\text {trans }} G_{\mathrm{a}} \alpha i_{\mathrm{ref} d}^{2} G_{\mathrm{is}} r^{2}+2 i_{\mathrm{dc}} r^{2} \alpha-2 K_{\mathrm{trans}} G_{\mathrm{a}}^{2} E_{s d} G_{\mathrm{is}} \alpha i_{\mathrm{ref} d} r v_{\mathrm{dc}} \\
& -2 K_{\mathrm{trans}} G_{\mathrm{a}} \alpha_{2} i_{\mathrm{ref} d} E_{s d} r L-v_{\mathrm{dc}}^{2} K_{\mathrm{trans}} G_{\mathrm{a}}^{3} G_{\mathrm{is}} i_{\mathrm{ref} d}^{2} w^{2} L^{2} \alpha \\
& +v_{\mathrm{dc}}^{2} K_{\mathrm{trans}} G_{\mathrm{a}}^{3} E_{s d} w^{2} L^{2} \alpha i_{\mathrm{ref} d}+2 i_{\mathrm{dc}} r L \alpha^{2} \\
& +K_{\mathrm{trans}} G_{\mathrm{a}}^{2} E_{s d}^{2} L v_{\mathrm{dc}} \alpha^{2}+2 K_{\mathrm{trans}} G_{\mathrm{a}}^{2} E_{s d}^{2} r v_{\mathrm{dc}} \alpha \\
& -K_{\mathrm{trans}} G_{\mathrm{a}} \alpha i_{\mathrm{ref} d} E_{\mathrm{sd}} r^{2}
\end{aligned}
$$

$$
\begin{aligned}
a_{0}= & K_{\text {trans }} G_{\mathrm{a}}^{2} E_{s d}^{2} r v_{\mathrm{dc}} \alpha^{2}+v_{\mathrm{dc}}^{2} K_{\mathrm{trans}} G_{a}^{3} E_{s d}(w L)^{2} \alpha^{2} i_{\text {ref } d}+i_{\mathrm{dc}} r_{2} \alpha^{2} \\
& -K_{\mathrm{trans}} G_{\mathrm{a}} \alpha_{2} i_{\mathrm{ref} d} E_{s d} r^{2} v_{\mathrm{dc}}
\end{aligned}
$$

\section{Appendix B. Parameters of the converters}

The parameters for the experimental and simulated VSC converter with current control are: the $A C$ voltage $E_{\mathrm{S}}=200 \mathrm{~V}$, $r=0.037 \Omega, L=3 \mathrm{mH}, C=2.2 \mathrm{mF}$, the current controller parameters are: $k_{\mathrm{p}}=13.3262, k_{i}=2.96 \times 10^{4}$. 
The DC-DC converter has power rate of $5 \mathrm{~kW}$ and the parameters are: $L_{1}=4 \mathrm{mH}, C_{\mathrm{f} 1}=0.3 \mathrm{mF}$, the current controller gains for parallel PI are $k_{\mathrm{p} 1}=2 \times 10^{-3}, k_{i 1}=1.0213$, the voltage controller gains are $4.19 \times 10^{-2}$, and $k_{i 2}=3.476$.

The wind turbine and photo-voltaic system have been designed to operate at nominal power at $10 \mathrm{~m} / \mathrm{s}$ of wind speed, and $S=1000 \mathrm{~W} / \mathrm{m}^{2}$ respectively. The battery storage system is rated at $3 \mathrm{~kW}$ and uses a bidirectional DC-DC converter with a capacitor $C_{\mathrm{b}}=0.18 \mathrm{mF}$, an inductance $L_{\mathrm{b}}=20 \mathrm{mH}$, the controller gains for the current loop are $k_{\mathrm{pb}}=106.609, k_{\mathrm{b}}=2.368 \times 10^{5}$, the voltage controller has the gains $k_{\mathrm{pb} 2}=41.6 \times 10^{-3}$, and $k_{i \mathrm{~b} 2}=4.626$.

\section{References}

[1] Kundur P. Power system stability and control. United States of America: McGraw-Hill; 1993.

[2] Liu J, Feng X, Ye Z, Lee FC, Borojevich D. Stability monitoring using voltage perturbation for dc distributed power systems. J Vib Control 2002;8:277-88.

[3] Liu J, Feng X, Lee F, Borojevich D. Stability margin monitoring for dc distributed power systems via current/voltage perturbation. In: Applied power electronics conference and exposition, 2001. APEC 2001. Sixteenth annual IEEE, vol. 2; 2001. p. 745-51.

[4] Feng X, Ye Z, Lee FC, Borojevich D. PEBB system stability margin monitoring. J Vib Control 2002;8:261-76.

[5] Danielsen S, Molinas M, Toftevaag T, Fosso OB. Constant power load characteristic's influence on the low-frequency interaction between advanced electrical rail vehicle and railway traction power supply with rotary converters. In: Modern electric traction, 2009 2009. p. 1-6.

[6] Areerak K, Bozhko S, Asher G, De Lillo L, Thomas D. Stability study for a hybrid ac-dc more-electric aircraft power system. IEEE Trans Aerospace Electronic Systems 2012;48(1):329-47. http://dx.doi.org/10.1109/TAES.2012.6129639.

[7] Areerak K-N, Bozhko S, Asher G, Thomas D. Dq-transformation approach for modelling and stability analysis of ac-dc power system with controlled PWM rectifier and constant power loads. In: Power electronics and motion control conference, 2008. EPE-PEMC 2008. 13th. 2008. p. 2049-54. http://dx.doi.org/ 10.1109/EPEPEMC.2008.4635567.

[8] Sun J. Small-signal methods for ac distributed power systems-a review. IEEE Trans Power Electron 2009;4(11):2545-54.

[9] Sun J, Karimi KJ. Small-signal input impedance modeling of line-frequency rectifiers. IEEE Trans Aerosp Electron Syst 2008;44(4):1489-97.

[10] Sun J, Chen M. Analysis and mitigation of interactions between PFC converters and the ac source. In: Power electronics and motion control conference, 2004. IPEMC 2004. p. 99-104.
[11] Sun J, Chen M. Low-frequency input impedance modeling of boost single phase PFC converters. IEEE Trans Power Electron 2004;22(4):1402-9.

[12] Sun J. AC power electronic systems: stability and power quality. In: Control and modelling for power electronics, 2008. COMPEL 2008. 11th workshop on 2008. p. $1-10$.

[13] Cespedes M, Sun J. Renewable energy systems instability involving gridparallel inverters. In: Applied power electronics conference and exposition 2009 2009. p. 1971-7.

[14] Belkhayat M. Stability criteria for ac power systems with regulated loads. Ph.D. thesis. Purdue University; 1997.

[15] Papathanassiou S, Hatziargyriou N, Strunz K. A benchmark low voltage microgrid network. In: CIGRE symposium 2005. p. 1-8.

[16] Girinon S, Baumann C, Piquet H, Roux N. Analytical modeling of the input admittance of an electric drive for stability analysis purposes. Eur Phys J 2009;47(1):1-8.

17] Harnefors L, Bongiorno $M$, Lundberg S. Input-admittance calculation and shaping for controlled voltage-source converters. IEEE Transactions on Industrial Electronics 2007;54(6):3323-34. http://dx.doi.org/10.1109 TIE.2007.904022.

[18] Khalil HK. Nonlinear systems. 2nd ed. United States of America: Prentice Hall; 1996.

[19] Gelb A, Vander Velde WE. Multiple-input describing functions and nonlinea system design. United States of America: McGraw-Hill; 1968.

[20] Mohan N, Undeland TM, Robbins WP. Power electronics, converters, applications, and design. 3rd ed. United States of America: John Willey \& Sons; 2003.

[21] Mohan N. First course on power electronics and drives. United States of America: MNPERE; 2003.

[22] Acevedo S, Giraldo E, Trejos E. Evaluation of power extraction to linear gain scheduling controllers in a small wind energy conversion system. In: Electronics, robotics and automotive mechanics conference (CERMA), 20102010. p. 557-62.

[23] Leonhard W. Control of electrical drives. 3rd ed. United States of America: Springer; 2001.

[24] Liu X, Wang P, Loh PC. A hybrid ac/dc microgrid and its coordination control. in press. IEEE Transactions on Smart Grid 2011:1-9. http://dx.doi.org 10.1109/TSG.2011.2116162.

[25] Barote L, Marinescu C. Renewable hybrid system with battery storage for safe loads supply. In: PowerTech, 2011 IEEE Trondheim 2011. p. 1-5. http:/ dx.doi.org/10.1109/PTC.2011.6019274

[26] Tremblay O, Dessaint L-A, Dekkiche A-I. A generic battery model for the dy namic simulation of hybrid electric vehicles. In: Vehicle power and propulsion conference, 2007. VPPC 2007. IEEE. 2007. p. 284-9. http://dx.doi.org/10.1109/ VPPC.2007.4544139.

[27] Guerrero J, Vasquez J, Matas J, de Vicuna L, Castilla M. Hierarchical control of droop-controlled ac and dc microgrids; a general approach toward standardization. IEEE Transactions on Industrial Electronics 2011;58(1):158-72. http://dx.doi.org/10.1109/TIE.2010.2066534. 